\title{
Trasplante combinado hepato-renal: a propósito de un caso
}

\author{
Jorge Roque $\mathrm{E}^{1}$, Gloria Ríos $\mathrm{M}^{1}$, Juan $\mathrm{Hepp}^{1}$, \\ Franco Innocenti $C^{1}$, Roberto Humeres $A^{1}$, \\ José M Palacios J ${ }^{1}$, Horacio Ríos $\mathbf{R}^{1}$, Leopoldo Suárez P1, \\ María Angélica Contreras $\mathrm{M}^{2}$, Montserrat Rius $\mathrm{A}^{1 \mathrm{a}}$.

\section{Combined liver-kidney transplantation. Report of one case}

\begin{abstract}
End stage renal disease is not an absolute contraindication for liver transplantation (LT) in patients with end stage liver disease. Actuarial patient and graft survival are comparable for children and adults who undergo LT alone and liver-kidney transplantation (LKT). The most common indications for LKT are the primary hyperoxaluria type I (PH1) and the liver and renal polycystic disease. We report a 12 years old boy with congenital hepatic fibrosis with severe portal hypertension, encephalopathy and polycystic kidney disease with end stage renal disease on dialysis that underwent LKT. During the second postoperative week, he had a biopsy-proven acute liver and renal rejection, that had a good response to corticosteroids. Thirty days after surgery, the liver biopsy was without rejection. No other complications were observed (Rev Méd Chile 2003; 131: 1309-12).
\end{abstract}

(Key Words: Kidney transplantation; Liver transplantation; Transplantation)

Recibido el 4 de junio, 2003. Aceptado el 17 julio, 2003.

${ }^{1}$ Unidad Trasplante Clínica Alemana de Santiago de Chile y ${ }^{2}$ Hospital Roberto del Río.

aEnfermera Universitaria, Clínica Alemana de Santiago de Chile.

$\mathrm{D}$ esde que el primer trasplante combinado hepato-renal (TCHR) fue comunicado por Margreiter y cols ${ }^{1}$, ha llegado a ser claro que la insuficiencia renal ya no es una contraindicación para realizar trasplante hepático (TH). La sobrevida de los injertos y hospederos es comparable a la del $\mathrm{TH}$ aislado tanto en adultos ${ }^{2-5}$ como en niños ${ }^{6,7}$. Las indicaciones para realizar TCHR son enfermedad hepática y renal terminal o enfermedad terminal de uno de estos órganos y disfunción grave e irreversible del otro ${ }^{6}$, siendo las

Correspondencia a: Dr. Jorge Roque E. Avenida Manquehue 1410, piso 1, Vitacura. Fax: 2124380.

E-mail: j.roque@entelchile.net principales causas hiperoxaluria primaria tipo 1 (HP1 $^{8-10}$ y enfermedad poliquística ${ }^{10-12}$. Algunos datos sugieren que el injerto hepático puede proteger del rechazo al injerto renal concomitantemente trasplantado $3,10,13,14$ y si bien se han esbozado algunas teorías sobre el origen de este fenómeno, también se han publicado opiniones al contrario $^{15,16}$. Nuestro centro ha comunicado previamente su experiencia en $\mathrm{TH}^{17,18}$, el objetivo de esta publicación es comunicar el caso de un niño portador de una enfermedad renal terminal y enfermedad hepática con hipertensión portal grave que fue sometido a TCHR con excelente resultado y revisar el estado actual del TCHR en la literatura. 


\section{CASO CĹNICO}

Se presenta el caso de un niño de 13 años con desnutrición crónica secundaria (bajo el percentil 5 para la edad en peso y talla), controlado desde el año de edad por enfermedad renal poliquística (ERP), con insuficiencia renal crónica terminal en hemodiálisis de un año de duración y fibrosis hepática congénita (FHC) con hipertensión portal grave, esplenomegalia y varices esofágicas esclerosadas; hospitalizado 3 veces en el último año por sepsis grave de origen respiratorio y biliar con descompensaciones graves de la síntesis hepática acompañadas de encefalopatía y ascitis. Fue sometido a trasplante simultáneo hepato-renal del mismo donante cadáver, crossmatch negativo. La inmunosupresión fue en base a esteroides, Micofenolato Mofetil (Cellcept ${ }^{\circledR}$, Roche, Pharmaceuticals, Nutley NJ, USA), Daclizumab (Zenapax ${ }^{\circledR}$, Roche Pharmaceuticals, Nutley, NJ, USA), y Tacrolimus (Prograf ${ }^{\circledR}$, Fujisawa USA, Deerfield, IL USA). Presentó durante la segunda semana rechazo simultáneo hepático moderado a severo y renal leve (ambos con biopsia percutánea compatible) con buena respuesta a esteroides. Sin otras complicaciones, fue dado de alta 20 días después del TCHR. Biopsia hepática a los 30 días sin rechazo. Cinco meses después se encontraba asintomático, asistiendo al colegio, con inmunosupresión basada en Tacrolimus y esteroides en dosis decrecientes y con pruebas sanguíneas de función hepática y renal normales.

\section{DisCUSIÓN}

Según datos de la Organ Procurement and Transplantation Network entre 1988 y 2002 se habían realizado en Estados Unidos 1.247 TCHR, de los cuales 43 (3\%) correspondían a pacientes entre los 11 y 17 años de edad ${ }^{19}$. Las causas más frecuentes para trasplante renal en pacientes con enfermedad hepática terminal incluyen enfermedad poliquística, nefropatía por oxalato, glomerulonefritis crónica, nefropatía diabética y toxicidad por ciclosporina $^{6,10}$. En adultos sometidos a TCHR con enfermedad hepática terminal y síndrome hepatorrenal se ha comunicado que tendrían la misma sobrevida que aquellos sometidos a $\mathrm{TH}$ aislado, sugiriendo que el manejo de ellos debiera ser sólo con TH aislado y una terapia inmunosupresora que limitara el uso de inhibidores de los calcineurínicos para minimizar el daño renal secundario $4,11,12$ Jeyarajah et al ${ }^{10}$ afirman que sólo la HP1 y la enfermedad poliquística hepática y renal son patologías que pueden razonablemente tratarse con TCHR. Esto tiene mucha importancia si se considera la escasez de donantes y por lo tanto el uso juicioso que debemos hacer de los órganos procurados.

En los niños los reportes se han enfocado en HP1 ${ }^{8-10}$. Estos pacientes nacen con una deficiencia hepática de la enzima glicoxilato-aminotransferasa, provocando una acumulación en el organismo de oxalato y glicolato. Los altos niveles de oxalato en el riñón precipitan y se depositan en el tejido, provocando nefrocalcinosis y evolucionando hacia la insuficiencia renal terminal en $90 \%$ de los casos antes de los 20 años $^{10}$. El TCHR permite corregir la deficiencia enzimática en el hígado. Los resultados del Registro Europeo de Trasplante de HP1 entre 1984 y 1997 muestran una sobrevida a 1, 2 y 5 años de $88 \%, 80 \%$ y $72 \%$, siendo la sobrevida para el injerto hepático en los mismos períodos de $82 \%$, $78 \%$ y $62 \%$ respectivamente ${ }^{8}$. Los pacientes trasplantados con menos de 2 años de diálisis previa tendrían mejor sobrevida ${ }^{8,9}$.

La Enfermedad Poliquística Hepatorrenal incluye un grupo diverso de anomalías que se caracterizan por ser hereditarias, cursar con quistes hepáticos, que pueden presentarse tardíamente y enfermedad renal quística. Se distingue una variedad autosómica dominante usualmente considerada como forma de presentación del adulto y una recesiva que es más frecuente en la infancia. Existe controversia sobre cuántas diferentes anomalías son englobadas en esta definición, la lesión hepática básica de todas las enfermedades fibroquísticas hepáticas es la malformación de la placa ductal, afectándose distintos niveles del árbol biliar intrahepático, que se manifiestan por quistes biliares asociados a fibrosis, provocando una hipertensión portal grave pero con función de síntesis relativamente bien conservada ${ }^{20}$. Nuestro paciente cursaba con ERP que se inició en la infancia y que lo llevó a insuficiencia renal terminal, requiriendo diálisis durante el último año y compromiso hepático complejo caracterizado básicamente por hipertensión portal, colangitis y encefalopatía como manifestación de su FHC. La 
posibilidad de realizar un procedimiento de derivación portal estaba muy limitada por tener una falla renal, diálisis dependiente y descompensaciones agudas frecuentes de la síntesis hepática, con encefalopatía grave y ascitis, por lo que requería un TCHR.

La FHC y ERP en la serie de TCHR pediátrico publicada por Grewal et $\mathrm{al}^{6}$ eran la primera causa con 5 casos entre 12 pacientes, con una sobrevida de los mismos 5 casos a 4,4 años de $80 \%$. La FHC aislada en otra serie de TCHR publicada por Reyes et $\mathrm{al}^{7}$ era la segunda causa (2 casos de 12 pacientes) luego de la oxalosis (4 casos de 12 pacientes), siendo allí las 2 primeras causas de enfermedad renal terminal la oxalosis y la ERP.

En niños sometidos a TCHR se comunica una sobrevida similar a la del TH aislado (a 5 años $67 \%$ versus $69 \%)^{6}$, mientras que Reyes et $\mathrm{al}^{7}$ en TCHR pediátrico comunican una sobrevida del hospedero a uno y 5 años de $66 \%$ y 58\%, respectivamente. En ambas series, la pérdida del injerto hepático no fue principalmente debido a una falla del mismo, sino por complicaciones que determinaron mortalidad como la sepsis, primera causa de muerte precoz $z^{2,3,6,7,11,14}$. Un elemento que se asoció al peor pronóstico en el TCHR fue la condición clínica del paciente previa al trasplante. Esto se reflejó en el status UNOS (United Network for Organ Sharing) ${ }^{6,7,10}$, ya que la sobrevida fue significativamente menor en aquellos pacientes que, por su peor estado clínico, requirieron ingreso a una unidad de cuidados intensivos previo al TCHR (status UNOS 1) que en los que tenían status UNOS 2 y 3 (sobrevida de pacientes a 5 años status UNOS $125 \%$ versus $88 \%$ status UNOS 2 y 3). Al momento del TCHR nuestro paciente se encontraba estable y sin requerimientos de cuidados intensivos.

Hay datos que sugieren que el injerto hepático tendría un efecto protector sobre el injerto renal concomitantemente trasplantado. Rasmussen et

\section{REFERENCIAS}

1. Margreiter R, Kramar R, Huber C. Combined liver and kidney transplantation. Lancet 1984; 1: 1077.

2. Gonwa T, Nery J, Husberg B Kuntmalm G. Simultaneous liver and renal transplantation in man. Transplantation 1988; 46: 690. $\mathrm{al}^{3}$ estudiaron 21 pacientes sometidos a TCHR y encontraron un aumento en la sobrevida del injerto renal comparado con aquellos sometidos a trasplante renal aislado (TRA). También observaron que hubo una significativa disminución de la tasa de rechazo celular agudo del injerto renal en pacientes sometidos a TCHR comparados con aquellos que recibieron TRA (9,5\% versus 37,2\%), no perdiéndose ningún injerto renal en el grupo de TCHR. Opelz et al ${ }^{13}$ comunican un estudio que utilizó datos del International Collaborative Transplant Study, encontrando que la sobrevida a largo plazo de los injertos renales en recipientes de TCHR era más alta que la de los pacientes sometidos a TRA. Interesantemente en este estudio se encontró que el injerto renal en el trasplante combinado riñón-corazón también tenía una sobrevida tan buena como la del grupo de TCHR. En contraste, un estudio realizado por Katznelson y Cecka ${ }^{15}$ usando la base de datos de la UNOS comparó 248 pacientes sometidos a TCHR versus 206 recipientes del riñón contralateral. Encontraron que la sobrevida del injerto renal a 3 años fue mejor en el grupo de TRA (80\% versus 68\%). Cuando excluyeron los pacientes fallecidos como causal de pérdida del riñón, las tasas de sobrevida a 3 años fueron similares. Los autores concluyeron que el injerto hepático no protegía al injerto renal simultáneamente trasplantado de rechazo celular agudo, ni mejoraba la sobrevida a largo plazo del riñón. Si bien nuestro paciente presentó rechazo celular agudo de ambos injertos, esto fue muy precoz y sólo requirió esteroides para su regresión, sin evidencias posteriores de nuevo rechazo.

En resumen se presenta el caso de un paciente pediátrico con enfermedad renal terminal e hipertensión portal grave, quien fue sometido con éxito a un TCHR. La insuficiencia renal grave en un paciente con enfermedad hepática terminal ya no es una contraindicación para realizar el trasplante hepático ni viceversa.

3. Rasmussen A, Davies HFS, Jamieson NV, Evans DB, CALNE RY. Combined transplantation of liver and kidney from the same donor protects the kidney from rejection and improves kidney graft survival. Transplantation 1995; 59: 919.

4. Jeyarajah DH, Gonwa TA, McBride M, Testa G, AbBasoglu O, Husberg BS et al. Hepatorenal 
syndrome. Combined liver kidney transplants versus isolated liver transplant. Transplantation 1997; 64: 1760-5.

5. Kuem V, Ringe B, Frei U, Pichlmayr R. Single-center experience of combined liver and kidney transplantation. Clin transplant 1995; 9: 39.

6. Grewal HP, Brady L, Cronin DC, Loss GE, Siegel CT, Oswald K ET aL. Combined liver and kidney transplantation in children. Transplantation 2000; 70: 100-5.

7. Reyes J, Mazariegos G. Liver transplantation: current management. Surg Clin North Am 1999; 79: 163-89.

8. JAMIESON NV. The results of combined liver/kidney transplantation for primary hyperoxaluria (PH1) 1984-1997. The European PH1 transplant registry report. European PH1 transplantation Study Group. J Nephrol 1998; 11: Suppl 1: 36-41.

9. Gagnadoux MF, Lacaile F, Niaudet P, Revilon $Y$, Jouvet P, JAN D ET AL. Long term results of liverkidney transplantation in children with primary hyperoxaluria. Pediatr Nephrol 2001: 16; 946-50.

10. Jeyarajah DR, McBride M, Kuntmalm GB, Gonwa TA. Combined liver-kidney transplantation: what are the indications? Transplantation 1997; 64: 1091-6.

11. Jeyarajah DR, Gonwa TA, Testa G, Abbasoglu O, GoLDSTEIN R, HusBerg BS et aL. Liver and kidney transplantation for polycystic disease. Transplantation 1998; 66: 529-32.

12. Distant DA, Gonwa TA. The kidney in liver transplantation. J Am Soc Nephrol 1993; 4: 129-36.

13. Opeiz G, Margreiter R, Döhler B. Prolongation of long-term kidney graft survival by a simultaneous liver transplant: the liver does it, and the heart does it too. Transplantation 2002; 74: 1390-4.
14. Margreiter R, Steurer W, Spechtenhauser B, KönigSRAINER A. Kidney transplantation together another solid organ from the same donor, a single center progres report. Clin Nephrol 2000; 53: 38-43.

15. Katzneison S, CECKA M. The liver neither protects the kidney from rejection nor improves kidney graft survival after combined liver and kidney transplantation from the same donor. Transplantation 1996; 61: 1403-5.

16. Eid A, Moore SB, Weisner RH, DeGoey SR, Nielson A, KRом RAF. Evidence that the liver does not always protects the kidney from hyperacute rejection in combined liver-kidney transplantation across a positive lymphocyte crossmatch. Transplantation 1990; 50: 331-4.

17. Hepp J, Ríos H, Súrez L, Zaror M, Quiroga M, Rodríguez G ET AL. Trasplante hepático en adultos: casuística de Clínica Alemana de Santiago. Rev Méd Chile 2002; 130: 779-86.

18. Roque J, Ríos G, ValderRama R, Hepp J, Ríos $H$, SuÁreZ L ET AL. Hiperplasia nodular focal del hígado en niños y trasplante hepático. Rev Chil Pediatr 2003; 74: 60-3.

19. Organ Procurement and Transplantation Network. Data: Multiple Organ transplant in the US by Recipient Age. US Multi-Organ transplant Performed: January 1, 1988 - January 31, 2003. For Multiple Organ = Liver - Kidney. Based on OPT data as of april 11, 2003. (Accessed April 20, 2003, at www.optn.org/latestData/rptData.asp.)

20. Michael C Kew. Tumores y quistes hepáticos. En: Mark Feldman, Bruce F. Scharschmidt, Marvin H. Sleisenger, Ed. Enfermedades Gastrointestinales y Hepáticas. 6무 Edición. Buenos Aires: Editorial médica Panamericana, 2000; 1480-1. 